Morton, A. G. \& Broadbent, D. (1955). J. gen. Microbiol. 12, 248-258

\title{
The Formation of Extracellular Nitrogen Compounds by Fungi
}

\author{
BY A. G. MORTON AND D. BROADBENT \\ Imperial Chemical Industries Ltd., Butterwick Research Laboratories, \\ Welwyn, Hertfordshire
}

SUMMARY: The production of nitrogen compounds in the culture medium by Scopulariopsis brevicaulis and other fungi has been studied. The formation of extracellular nitrogen compounds accompanied the primary assimilation of ammonia, nitrate and organic nitrogen in all experimental conditions so far investigated among the various factors studied. The amount of these extracellular nitrogen compounds was affected markedly only by the supply of certain trace elements. It was lowest when the concentration of trace elements was high, and increased as the concentration of trace elements approached that limiting growth. Most of the extracellular nitrogen appeared to be peptide in nature, yielding some 14 amino acids on hydrolysis. The fungus was unable to assimilate the extracellular nitrogen compounds formed but assimilated the constituent amino acids when these were liberated by acid hydrolysis.

When fungi grow on inorganic nitrogen sources organic nitrogen compounds appear in the culture medium at the same time (Morton, 1951). This phenomenon seems to be frequent among micro-organisms; it has been observed in bacteria (Fedorov, 1952 ; Hotchkiss, 1950; Proom \& Woiwod, 1949), in bluegreen algae (Fogg, 1952; Henriksson, 1951; Watanabe, 1951) and in fungi by many authors (Dietzel, Behrenbruch \& Eucken, 1951; Hockenhull, 1950; Iwanoff \& Krupkina, 1929; Nielson \& Hartelius, 1937; Reindel \& Hoppe, 1952; Steinberg, 1939). The formation of extracellular nitrogen compounds by fungi always occurs during the period of active assimilation of nitrogen, as Nielson \& Hartelius (1937) noted in experiments with yeast. This link with primary nitrogen metabolism is the most significant feature of our observations and prompted further investigation. These secondarily formed organic nitrogen compounds which appear in the medium during growth will for the sake of brevity be termed simply 'extracellular nitrogen'.

\section{EXPERIMENTAL}

The fungi used were grown in surface culture in small flasks containing $20 \mathrm{ml}$. of culture medium of the following basal composition: $50 \mathrm{~g}$. glucose; $1.0 \mathrm{~g}$. $\mathrm{KH}_{2} \mathrm{PO}_{4} ; 0.5 \mathrm{~g} . \mathrm{MgSO}_{4} .7 \mathrm{H}_{2} \mathrm{O}$; trace elements $(200 \mu \mathrm{g}$. Fe, $38 \mu \mathrm{g}$. Cu, $225 \mu \mathrm{g}$. $\mathrm{Zn}$, 24 $\mu \mathrm{g}$. Mn, and $40 \mu \mathrm{g}$. Mo); water to 11 . Different nitrogen sources were added as required. The nitrogen source was always sterilized separately from the other components of the medium in order to avoid any interaction with the carbohydrate present. The $\mathrm{pH}$ value of the media was adjusted to 6.5 initially, and the cultures were incubated at $25^{\circ}$. 
Replicate flasks were harvested at intervals after inoculation. The culture medium was gently washed out, made up to standard volume to allow for evaporation, and filtered to remove mycelium and spores. Estimations of ammonia and nitrate were carried out on Conway micro-diffusion units by methods previously detailed (Morton \& MacMillan, 1954). Total nitrogen was determined by micro-Kjeldahl. Amino nitrogen was determined as ammonia liberated by the action of ninhydrin (MacFadyen, 1944). The ammonia was estimated by Nessler's reagent after vacuum distillation following the method of Archibald (1943).

For most of the experiments a strain of Scopulariopsis brevicaulis (Sacc.) Bainier (No. 55/OS/A 1) was used. Other species used were Aspergillus niger v. Tieghem, Penicillium chrysogenum Thom, Trichoderma viride Pers. ex Fries, Botrytis allii Munn.

\section{RESULTS}

\section{General features of nitrogen assimilation}

The normal course of development in Scopulariopsis brevicaulis is illustrated graphically in Fig. 1 where the results from a number of separate experiments are assembled. In all experiments $6 \cdot 6 \mathrm{mg}$. $\mathbf{N}$ (as ammonium sulphate)/flask

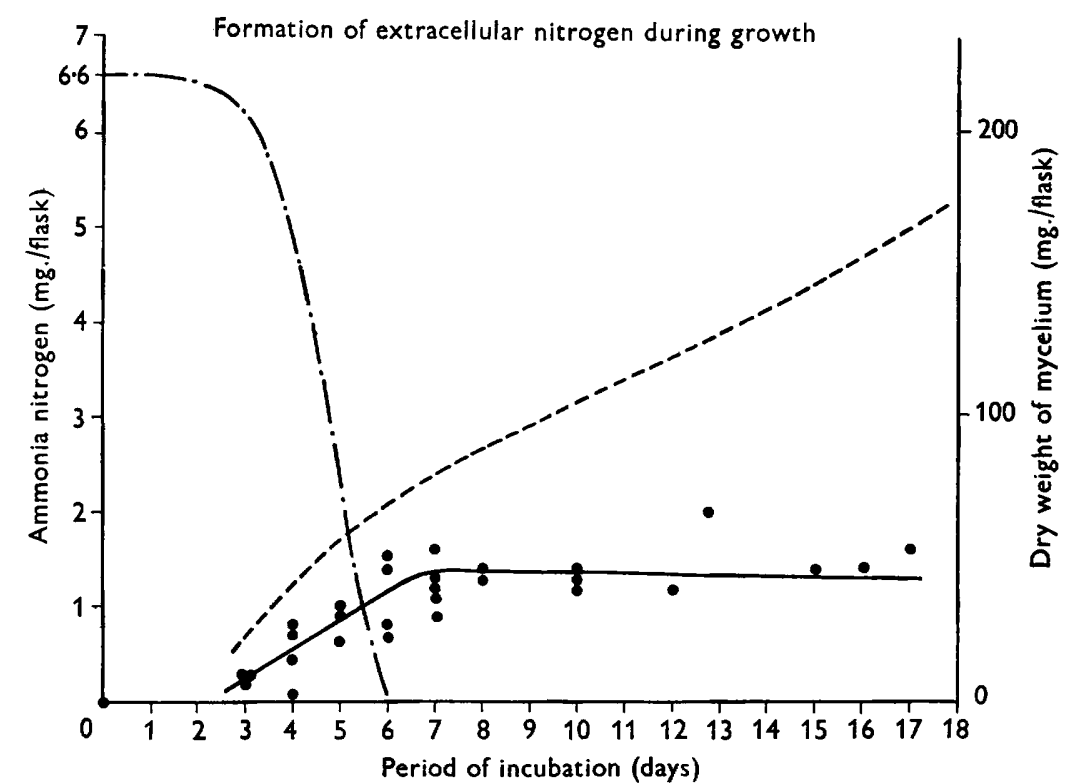

Fig. 1. Formation of extracellular nitrogenous material during growth (ammonia as N source). Dry weight - - - ; ammonia nitrogen, .-.-.; extracellular nitrogen, - -

was present initially. The main carbon source was glucose, but $1 \%(\mathrm{w} / \mathrm{v})$ sodium malate, succinate or citrate was added to prevent adverse $\mathrm{pH}$ changes (see Morton \& MacMillan, 1954). It will be seen that 'organic' nitrogen in the medium rose rapidly from about the $3 \mathrm{rd}$ to the 6 th or 7 th day, precisely during 
the time when ammonia-nitrogen was most rapidly disappearing. After exhaustion of the ammonia the concentration of 'organic' nitrogen remained constant (within the limits of experimental error) up to 20 days and even longer.

The amount of glucose in the basal medium was more than sufficient to allow assimilation of all the nitrogen supplied. Indeed the dry weight of the fungus increased steadily at the expense of glucose for some time after all the ammonia had disappeared. The dry weight of mycelium roughly doubled itself between 7 and 20 days. There was no evidence of autolysis (recognized by the re-appearance of free ammonia in the medium) in this period. Microscopic examination showed that this secondary increase in dry weight was due to new growth and not simply to an accumulation of materials in the mycelium already formed. This growth was not the expression of conidia formation alone, although the conidia were mainly produced at this stage; there was, however, a considerable formation of new vegetative hyphae.

Since no measurable quantity of the extracellular organic nitrogen was used, it seems clear that there must be extensive re-mobilization of the internal nitrogen of the mycelium during this secondary growth period. Evidence of such re-mobilization was seen in the appearance of the mycelium. The cytoplasm in the older hyphae became highly vacuolate, whilst new hyphae grew out and were narrower in diameter and filled with dense scarcely vacuolate cytoplasm.

Thus, in normal cultural conditions, when glucose supply was not limiting, at least two distinct phases of nitrogen metabolism were observed. The first phase involved the assimilation of the initially added ammonia, and was accompanied by the formation of extracellular nitrogenous compounds. The second phase, judging by the mass of new hyphae formed, was an extensive re-mobilization of the internal nitrogen of the mycelium, accomplished without measurable quantitative change in the extracellular nitrogenous material already formed. There was no detectable mobilization of extracellular nitrogen, although glucose and other constituents of the culture medium were present in adequate amounts, as demonstrated by the fact that further additions of ammonia or nitrate were readily assimilated. The possibility of a balanced turn-over of extracellular nitrogen is not excluded, however, although it seems unlikely.

\section{Quantitative aspects}

The formation of extracellular nitrogenous material has been observed in a number of fungi and appears to occur generally. It is apparent from Tables 1 and 2 that the proportion of initially available nitrogen converted into extracellular nitrogen varied with the fungal species and the nature of the nitrogen source. The amount of extracellular nitrogen may represent a surprisingly high proportion of the nitrogen supplied initially. In Scopulariopsis brevicaulis 20-25\% of the nitrogen was finally found in this extracellular form whether the nitrogen source was ammonia or nitrate. Experiments were therefore carried out to see whether the concentration of extracellular nitrogen could be altered by changes in nutritional or other environmental conditions. 
Table 1. Amount of nitrogen assimilated which appears in the medium after 7 days growth

$\begin{array}{lcc}\text { Fungus } & \begin{array}{c}\text { Nitrogen source } \\ \text { (in basal medium) }\end{array} & \begin{array}{c}\text { Extracellular N } \\ \text { (as \% initially } \\ \text { added N) }\end{array} \\ \text { Aspergillus niger } & \text { Ammonia } & \mathbf{7 \cdot 5} \\ & \text { Nitrate } & \mathbf{3 \cdot 5} \\ \text { Penicillium chrysogenum } & \text { Ammonia } & \mathbf{2 0 \cdot 0} \\ & \text { Nitrate } & \mathbf{7 \cdot 5} \\ \text { Trichoderma viride } & \text { Ammonia } & \mathbf{2 7 \cdot 0} \\ & \text { Nitrate } & \mathbf{3 6 \cdot 0} \\ \text { Botrytis alii } & \text { Ammonia } & \mathbf{2 3 \cdot 5}\end{array}$

Table 2. Formation of extracellular nitrogen in relation to initially added nitrogen which disappeared

\begin{tabular}{|c|c|c|c|c|}
\hline & & Amoun & f $\mathbf{N}$ supplied & g./flask) \\
\hline & & $6 \cdot 6$ & $13 \cdot 2$ & $6 \cdot 6+6 \cdot 6$ \\
\hline Fungus & $\begin{array}{c}\text { Nitrogen } \\
\text { source }\end{array}$ & Extracel & ar $\mathbf{N}$ as $\% \mathbf{N}$ & similated \\
\hline copulariopsis brevicaulis & Ammonia & $25 \cdot 35 \pm 5 \cdot 58$ & $25 \cdot 80 \pm 2 \cdot 86$ & \\
\hline Penicillium ariceofulrum & Nitrate & $20 \cdot 03 \pm 4.47$ & $16 \cdot 62 \pm 2 \cdot 08$ & $16 \cdot 50 \pm 1 \cdot 98$ \\
\hline Penicillium griseofulvum & Nitrate & $13 \cdot 00 \pm 3 \cdot 55$ & $12 \cdot 50 \pm 0.70$ & $12 \cdot 34 \pm 2 \cdot 79$ \\
\hline
\end{tabular}

Change in concentration of nitrogen supplied. The effect of different nitrogen concentrations in the culture medium was tested. The amount of nitrogen in the culture medium was doubled, the concentration of glucose and salts being raised sufficiently to ensure complete assimilation of the extra nitrogen. In some experiments twice the usual concentration of nitrogen (as ammonia or nitrate) was added initially. In other experiments the fungus was grown on basal medium with the usual amount of ammonia or nitrate until this was just exhausted (6-7 days); then a second quantity of nitrogen compound was added, and growth allowed to continue until all the added nitrogen had again disappeared. The results of several experiments are summarized in Table 2. The amount of extracellular nitrogen formed is seen to be almost directly related to the amount of nitrogen assimilated, irrespective of whether the nitrogen was presented in one or in two doses.

In some other experiments the glucose concentration was decreased to $0.5 \%(w / v)$, leaving the nitrogen source at its usual concentration. With this smaller concentration of carbohydrate Scopulariopsis brevicaulis was able to assimilate only two-thirds of the added nitrogen (ammonia) in the medium, yet $15 \%$ of the nitrogen assimilated appeared as extracellular nitrogen. These results emphasize the close relation which exists between primary nitrogen uptake and the formation of extracellular nitrogen.

Nature of carbon source. Since glucose can form compounds with ammonia fairly readily it seemed possible that the formation of extracellular organic nitrogen might be related to the presence of glucose. However, when glucose 
was replaced by $5 \%$ sodium acetate (known to be a good carbon source for Scopulariopsis brevicaulis), the nitrogen (ammonia) in the medium was completely assimilated and the usual proportion of it (18\%) appeared as extracellular nitrogen.

Growth factors and inhibitors. Scopulariopsis brevicaulis was grown with and without a vitamin supplement containing biotin, thiamine, pyridoxin, nicotinic acid and inositol. The amount of extracellular nitrogen formed was exactly the same in both series.

The effect of a number of inhibitors and antibiotics was tested, including iodoacetate, coumarin, griseofulvin, aurantiogliocladin, whilst in other experiments growth was affected by diminishing the amount of sulphur or phosphorus in the medium to concentrations at which they became limiting. The results will not be reported in detail since none of the treatments altered significantly the proportion of extracellular nitrogen to nitrogen assimilated, although in several experiments the actual amount of nitrogen assimilated was considerably decreased.

Volume of culture medium. The proportion of extracellular nitrogen formed was not significantly altered by dissolving the basal amount of nitrogen source and other components of the medium in one half and in twice the usual volume of fluid. The fungus was grown in submerged (shaken) culture in order to test the effect of dilution with the least possible complication from variation in surface/volume ratio. The proportion of extracellular nitrogen to initially added nitrogen was practically the same in shaken and in surface culture.

Trace elements. The basal medium was normally supplemented by the addition ( $1 \mathrm{ml} . / \mathrm{l}$.) of a solution containing the trace elements iron, copper, zinc, manganese and molybdenum, at concentrations which experience indicated to be adequate for the growth of most fungi. In some experiments the concentration of trace elements was varied by adding multiples of the normal amounts. The effects on the formation of extracellular nitrogen (in a nitrate medium) are shown in Table 3.

Table 3. Formation of extracellular nitrogenous material by Scopulariopsis brevicaulis with nitrate as added $\mathbf{N}$ source and with different concentrations of trace element mixture

\begin{tabular}{|c|c|c|c|c|}
\hline \multirow{2}{*}{$\begin{array}{l}\text { Trace element } \\
\text { addition }\end{array}$} & \multicolumn{2}{|c|}{$\begin{array}{c}\text { Extracellular nitrogen } \\
\text { (as \% initially added } \\
\text { nitrogen which disappeared) }\end{array}$} & \multicolumn{2}{|c|}{$\begin{array}{l}\text { Dry matter (mg.) at } \\
10 \text { days (all } \mathrm{NO}_{3} \text { taken } \\
\text { up from medium) }\end{array}$} \\
\hline & Expt. 1 & Expt. 2 & Expt. 1 & Expt. 2 \\
\hline No addition & $32 \cdot 0$ & $26 \cdot 0$ & 181 & 198 \\
\hline Normal dose* & $16 \cdot 0$ & 16.5 & 192 & 192 \\
\hline Normal dose $\times 2$ & $13 \cdot 0$ & . & 186 & - \\
\hline Normal dose $\times 10$ & $7 \cdot 0$ & $9 \cdot 0$ & 192 & 192 \\
\hline Normal dose $\times 20$ & . & $7 \cdot 0$ & . & 204 \\
\hline Normal dose $\times 50$ & . & $7 \cdot 0$ & . & - \\
\hline
\end{tabular}

* The normal dose of trace elements is equivalent to the addition of $200 \mu \mathrm{g}$. Fe, $38 \mu \mathrm{g} . \mathrm{Cu}$, $225 \mu \mathrm{g}$. $\mathrm{Zn}, 24 \mu \mathrm{g}$. Mn, and $40 \mu \mathrm{g}$. Mo, per litre of medium. 
The extracellular nitrogen was highest when no trace elements were added, when the fungus must rely on the amounts of various elements present as impurities in the constituents of the medium, coming from the glassware, or introduced with the inoculum. No special precautions to exclude trace elements were taken in these experiments. The amount of extracellular nitrogen was decreased by $40-50 \%$ by addition of the usual supplement of trace elements. The addition of 10 times the usual supplement caused a further decrease (c. $50 \%$ ) in the amount of extracellular nitrogen, but further additions had no effect.

Even at the lowest concentration of trace elements (no addition) all the nitrate nitrogen was completely assimilated, and there was no decrease in final dry weight of mycelium. The only evidence that the supply of trace elements might have been limiting was the fact that at the lower concentrations (no addition and normal dose) the rate of assimilation of nitrate and the rate of dry-matter accumulation were distinctly slower during the first 5 days of growth. There were no differences in rate of nitrate assimilation and dry-matter accumulation between cultures receiving 2 to 50 times the normal dose of trace elements. Further experiments will be required to determine the extent to which the effects on the yield of extracellular nitrogen are related to the concentration of individual trace elements. It is clear, however, that more extracellular nitrogen was formed when the supply of trace elements was decreased. On the other hand, even with relatively high concentrations of added trace elements the formation of extracellular nitrogen was not suppressed altogether. The possibility that some further trace element, not intentionally supplied, may be involved clearly requires investigation.

Organic nitrogen sources. The question arose whether extracellular nitrogen was produced when nitrogen was supplied initially in an organic form instead of as ammonia or nitrate. Scopulariopsis brevicaulis was grown on the basal medium plus various single amino acids, namely glycine, L-glutamic acid, L-arginine, L-alanine, $\gamma$-aminobutyric acid; enzyme-hydrolysed casein was also tested. Growth was vigorous on all amino acids tested except arginine, and the added single amino acids added disappeared, as shown by paper chromatography. Even with arginine, where growth was much slower, all this amino acid eventually disappeared. In every case nitrogen equivalent to $14-24 \%$ of that added initially was found in the medium, but this nitrogenous material was different in chemical nature from that supplied initially (see below) and evidently represented extracellular nitrogenous compounds formed during metabolism (growth). The use of amino acids as nitrogen source thus involved the formation of extracellular nitrogenous compounds just as when inorganic nitrogen sources (ammonia, nitrate) were used.

\section{The nature of the extracellular nitrogenous material}

The chemical nature of the extracellular nitrogenous material was investigated in culture fluid of Scopulariopsis brevicaulis, taken after 7 days growth when the concentration of nitrogen in the medium had become constant, and all the ammonia or nitrate added initially had disappeared. There 
were no differences in the general character of extracellular nitrogenous material whether the nitrogen source was ammonia, nitrate or an amino acid.

A large volume of culture filtrate was evaporated under reduced pressure to about one-eighth its original volume. No material was precipitated during this evaporation, or after treatment with trichloroacetic acid, phosphotungstic acid or other protein precipitants. Between 92 and $95 \%$ of the nitrogen was dialysable against running water in $48 \mathrm{hr}$. Not more than $5 \%$ of the extracellular nitrogenous material can therefore be protein in nature (extracellular enzymes).

The extracellular nitrogenous material gave reactions with ninhydrin for amino nitrogen suggesting the presence of free amino acids. Quantitative estimation of amino nitrogen showed that not more than $10 \%$ of the extracellular nitrogen could be present as the $\alpha$-amino groups of free amino acids. The remaining nitrogen $(80-85 \%$ of the total) did not react with ninhydrin directly. After hydrolysis with $\mathrm{N}-\mathrm{HCl}$ at $100^{\circ}$ for several hours, however, considerable quantities of amino nitrogen (reacting with ninhydrin) were liberated, which would suggest that peptide material was originally present. On estimation this liberated amino nitrogen was found to account for $60-80 \%$ of the nitrogen of this fraction. Some of the amino acids liberated on hydrolysis were basic and contained nitrogen not estimated by ninhydrin. After making reasonable allowance for this it seems probable that there was some nitrogen (perhaps $10-20 \%$ of this fraction) not to be accounted for as peptide.

Investigation of the nature of the extracellular nitrogenous material was carried further by paper chromatography. The presence of free amino acids in the culture fluid was confirmed and the main ones present at the early harvests (5-7 days) were identified as alanine, glutamic, aspartic and $\gamma$-aminobutyric acids. Traces of glycine, serine, proline, valine and leucine were also present and tended to become more distinct in later harvests (14-20 days). Rough estimates of the amounts of free amino acids were made from the chromatograms and confirmed that less than $10 \%$ of the extracellular nitrogen occurred as free amino acids.

Examination of the main (peptide) extracellular nitrogen fraction after hydrolysis shows the presence of 14 amino acids: alanine, glutamic acid, aspartic acid, $\gamma$-aminobutyric acid, glycine, serine, threonine, proline, arginine, histidine, lysine, valine, tyrosine, leucine, and (probably) $\beta$-alanine. The first three amino acids listed were present in slightly larger amounts relative to the others but there was no marked preponderance of any one amino acid. Little more can be said at present about this peptide fraction. When the unhydrolysed material was examined by paper chromatography certain slowmoving indistinct spots which reacted very faintly with ninhydrin appeared on the chromatogram. When these spots were cut out and hydrolysed they yielded a range of amino acids. Attempts to resolve the spots by running solvent for long periods were not conclusive, and it remains uncertain whether the peptide fraction represents mainly a single polypeptide or a group of closely related peptides which tended to move together on the chromatogram.

The amino acid composition of different samples of the peptide fraction, as 


\section{Extracellular nitrogen in fungal cultures}

far as it is possible to judge from chromatograms of the hydrolysate, shows little variation either in the number of amino acids present or in their relative proportions. No significant changes in composition were obvious either with time (7-20 days) in a given experiment, or with variations in cultural conditions (nitrogen source, carbon source, etc.).

\section{Nitrogen in the mycelium}

Some interest attaches to the question of the relation between the internal nitrogen of the mycelium and the extracellular nitrogenous material. Mycelium was extracted with a solution of $70 \%(v / v)$ ethanol in water. After concentration of the extract total- $\mathrm{N}$ and amino- $\mathrm{N}$ were determined, and the material examined by paper chromatography. Free amino nitrogen accounted for $44 \%$ of the total nitrogen extracted from the mycelium. Qualitatively there was a rather close relation between the free amino acids of the mycelial extract and the free amino acids of the extracellular nitrogenous material. Thus at 5 days the main amino acids in the mycelial extract were alanine, glutamic acid, aspartic acid and $\gamma$-aminobutyric acid, and these were the most prominent also in the culture medium. At later stages (14 days) several other amino acids appeared in the mycelial extract, although at much lower concentrations than the main ones, and the same acids were then detected in the culture medium. Quantitatively, however, there was a great difference between amounts of various amino acids in the mycelium and in the metabolism fluid; the ratio of the concentration of free amino acids in the mycelium to concentration in the metabolism fluid was $c$. 150:1 at 5 days and 100:1 at 14 days. When the material extracted by $70 \%(v / v)$ ethanol in water was hydrolysed there was an increase in free amino- $\mathrm{N}$ corresponding to $6-7 \%$ of the total $\mathbf{N}$. This presumably represented peptide nitrogen extracted in the given conditions.

\section{Physiology of the formation of extracellular nitrogenous material}

In the usual experimental conditions the extracellular nitrogenous material appeared not to be metabolized further when the carbohydrate supply and other conditions would seem to have been favourable. It was thus of interest to determine whether the fungus could utilize some or all of its own extracellular nitrogenous material in different conditions. Since Scopulariopsis brevicaulis grows readily with certain single amino acids as $\mathbf{N}$ source, its ability to use the free amino acids of the extracellular nitrogenous material was first examined. Culture fluid from a 15-day growth of $S$. brevicaulis was passed through a column of a sulphonated polystyrene resin which retained the free amino acids. These were then eluted from the resin and used as the nitrogen source (at the usual concentration of $N$ ) when added to the basal medium. $S$. brevicaulis grew well on this medium, obviously utilizing the nitrogen, and after 14 day growth only $24 \%$ of the nitrogen added remained in the medium; this amount did not differ significantly from the amount left when pure L-amino acids were used. 
It is clear that the free amino acids of the extracellular nitrogenous material (after concentration) could be used by the fungus. They only make up $10 \%$ of the extracellular nitrogen, however, whilst their concentration in the metabolism fluid was less than $1 / 100$ of the free amino-acid concentration in the mycelium (calculated from the fresh weight). Possibly this low concentration of amino acids in the metabolism fluid represented a leakage from the mycelium: in other words, the hyphal permeability was such that the concentration of amino acids in the medium could not be decreased below a certain value. The close qualitative relation found between the amino acids of the mycelial extract and of the metabolism fluid would be in agreement with this view.

To test assimilability of the peptide fraction, culture filtrate from 15-day Scopulariopsis brevicaulis was evaporated down to suitable volume at a low temperature, and was used as the nitrogen source (at the usual concentration of $6.6 \mathrm{mg}$. $\mathrm{N} /$ flask), in fresh culture medium; the free amino acids were not removed. One set of flasks was inoculated with $\boldsymbol{S}$. brevicaulis and others, for comparison, with Penicillium griseofulvum and Aspergillus niger. The culture filtrate was not toxic, as was shown by the excellent growth of all three fungi in flasks containing similar medium + potassium nitrate.

All three species made some growth when the extracellular nitrogenous material of Scopulariopsis brevicaulis was used as nitrogen source. The proportion of the initially-added nitrogen which was left in the culture medium after 15-20 days' incubation was $65 \%$ for $S$. brevicaulis, $58 \%$ for Penicillium griseofulvum, and $52 \%$ for Aspergillus niger. Some $10 \%$ of the initially added nitrogen was present as free amino acids, and could be assimilated. Therefore, of the peptide material between 25 and $40 \%$ of its nitrogen appeared to be assimilated in these conditions. These figures are only approximate since they neglect the formation of more extracellular nitrogenous material. It is not certain that some change in the extracellular peptide nitrogen, making it partly assimilable, did not take place during evaporation of old culture fluid and the preparation of the new medium containing it. Nevertheless, it seems probable that $50-60 \%$ of the extracellular nitrogen was not further metabolized by Scopulariopsis brevicaulis.

Finally the ability of the fungi to utilize the products of acid hydrolysis of extracellular peptide was tested. Culture filtrate from 15-day incubation of Scopulariopsis brevicaulis, after concentration, was hydrolysed for $18 \mathrm{hr}$. with $2 \mathrm{~N}-\mathrm{HCl}$ at $60^{\circ}$. The dark hydrolysate was treated with acetic acid-treated charcoal (no nitrogen removed) and after neutralization was used as nitrogen source in fresh culture medium. Flasks were inoculated with the same three fungi as before. Control flasks with acid hydrolysate + nitrate showed that the hydrolysate was not toxic.

All three fungi grew better than on unhydrolysed extracellular nitrogenous material; the proportion of initially added nitrogen which remained in the medium after growth was $\mathbf{4 0} \%$ for Scopulariopsis brevicaulis, and $34 \%$ for Penicillium griseofulvum and Aspergillus niger. Allowing for a fresh formation of extracellular nitrogenous material and for the occurrence of some non- 
peptide nitrogen, these figures suggest that the amino acids derived from the peptide material by acid hydrolysis were assimilated normally. Thus the nonassimilability of the peptide material cannot be ascribed to the presence in it of D-amino acids, but appears to depend on its general structure. This is analogous to the case of naturally occurring toxic peptides, where toxicity is bound up with total structure and not with the occurrence of occasional D-amino acids or specific chemical groups.

\section{DISCUSSION}

The experiments described emphasize the regular connexion that exists between the disappearance of the initial nitrogen compound added (e.g. ammonia, nitrate) and the formation of extracellular nitrogenous material by fungi, and show that this extracellular nitrogenous material is not simply a product of autolysis or senescence. The proportion of initially added nitrogen which reappeared in the culture medium remained unchanged over a wide range of experimental conditions but was significantly affected by the concentration of trace elements supplied. In none of our experiments was the concentration of trace elements low enough to limit the final dry weight of the fungus or to prevent the assimilation of all the nitrogen supplied. Nevertheless, when judged by the initial rate of nitrogen uptake and dry-matter increase it seems that the concentration of trace elements normally used was suboptimal. It is significant that extracellular nitrogen was formed in greatest amount when the supply of trace elements shows signs of becoming limiting, suggesting a diversion of nitrogen caused by nutritional deficiency or imbalance. Extracellular nitrogen continued to be formed, however, even in conditions when the concentration of trace elements seemed unlikely to be limiting.

In all the experimental conditions used the general chemical nature of the extracellular nitrogenous material formed remained the same. A small proportion consisted of free amino acids, but the bulk of the material was probably peptide in character. It gave rise to a number of amino acids on hydrolysis, and these accounted for $80-100 \%$ of the nitrogen of this fraction.

The presence of free amino acids in the culture medium during growth was noted by Mackenzie \& Cook (1951) for Penicillium notatum and by Rao \& Venkataraman (1952) for $\boldsymbol{P}$. chrysogenum. The latter authors found accumulation of amino acids at first, followed by their utilization during later stages of growth. They did not look for peptide nitrogen. Reindel \& Hoppe (1952) showed that extracellular nitrogenous material from yeast contained material which yielded 11 amino acids on hydrolysis, as well as free amino acids and unidentified nitrogen compounds. The proportion of free amino acids found $(\mathbf{5 0} \%$ ) was considerably higher than for $S$. brevicaulis. Reindel \& Hoppe found that not more than $20 \%$ of the extracellular nitrogen could be re-utilized by a fresh yeast inoculum. This is in agreement with our results with mould fungi.

It must be said that our experiments do not yet throw much light on the physiological significance of the extracellular nitrogenous material. The 
possibility has been considered that these nitrogen compounds may represent intermediates in protein synthesis which can only be utilized at a particular stage of growth and which tend to accumulate in certain conditions of nutritional imbalance. This interpretation does not seem very probable, however, in view of the evidence that this extracellular nitrogen is scarcely utilized by a fresh inoculum of the fungus even in a favourable medium. The problem seems most likely to be resolved by a parallel study of the changes in intracellular and extracellular nitrogen fractions during the course of nitrogen assimilation, especially in relation to the effects of the trace elements.

The authors wish to thank Miss Winifred F. Coulthard, Mr A. G. F. Dickerson, and Mr D. A. Terry for skilled technical assistance.

\section{REFERENCES}

Archibald, R. M. (1943). The estimation of ammonia in the presence of glutamine. J. biol. Chem. 151, 141.

Dietzel, E., Behrenbruch, H. \& Eucken, M. (1951). Stoffwechselversuche an Actinomyceten. Arch. Mikrobiol. 15, 179.

Fenorov, M. V. (1952). Partial diversion of the products of nitrogen assimilation by Azotobacter into the medium. Microbiology, Moscow, 21, 395.

FogG, G. E. (1952). The production of extra-cellular nitrogen substances by a bluegreen alga. Proc. roy. Soc. B, 139, 372.

Henriksson, E. (1951). Nitrogen fixation by a bacteria-free symbiotic Nostoc strain isolated from Collema. Physiol. Plant. 4, 542.

Hockenhuld, D. J. D. (1950). Studies in the metabolism of mould fungi. I. A preliminary study of the metabolism of carbon, nitrogen and sulphur in Aspergillus nidulans. J. exp. Bot. 1, 194.

HotchкISs, R. D. (1950). The abnormal course of bacterial protein synthesis in the presence of penicillin. J. exp. Med. 91, 351.

Iwanoff, N. N. \& Krupkina, F. A. (1929). Über die Stickstoffausscheidung der Hefe während der Gärung. Biochem. Z. 212, 255.

MACFADYEN, D. A. (1944). The determination of ammonia evolved from $\alpha$-amino acids by ninhydrin. J. biol. Chem. 153, $50 \%$.

Mackenzie, R. M. \& Cook, R. P. (1951). The nitrogen metabolism of Penicillium notatum. Biochem. J. 50, iii.

Morton, A. G. (1951). Formation of extra-cellular nitrogen compounds by fungi. Nature, Lond. 168, 333.

Morton, A. G. \& Macmillan, A. (1954). The assimilation of nitrogen from ammonium salts and nitrate by fungi. J. exp. Bot. 5, 232.

Nielson, N. \& Hartelius, V. (1937). Untersuchungen über die Stickstoffassimilation der Hefe. VIII. Untersuchungen über die Stickstoffabgabe der Hefe während des Wachstums. C.R. Lab. Carlsberg (sér. physiol.), 22, 24.

Proom, H. \& Worwod, A. J. (1949). The examination by paper chromatography of the nitrogen metabolism of bacteria. J. gen. Microbiol. 3, 319.

Rao, P. L. N. \& Venkataraman, R. (1952). Nitrogen metabolism of Penicillium chrysogenum. Experientia, 8, 350.

Reindel, F. \& Hoppe, W. (1952). Über die stickstoffhältigen Ausscheidungsprodukte der Hefe. Chem. Ber. 85, 716.

Steinberg, R. A. (1939). Optimum solutions as physiological reference standards in estimating nitrogen utilisation by Aspergillus niger. J. agric. Res. 58, 717.

Watanabe, A. (1951). Production in cultural solution of some amino acids by the atmospheric nitrogen-fixing blue-green algae. Arch. Biochem. Biophys. 34, 50. 